Acadiensis

Journal of the History of the Atlantic Region

Revue d'histoire de la région Atlantique

\title{
Une Acadie à construire . . . mais où?
}

\section{Clint Bruce}

Volume 47, numéro 2, été-automne 2018

URI : https://id.erudit.org/iderudit/1058011ar

DOI : https://doi.org/10.1353/aca.2018.0026

Aller au sommaire du numéro

Éditeur(s)

Department of History at the University of New Brunswick

ISSN

0044-5851 (imprimé)

1712-7432 (numérique)

Découvrir la revue

Citer cette note

Bruce, C. (2018). Une Acadie à construire ... mais où? Acadiensis, 47(2).

https://doi.org/10.1353/aca.2018.0026 d'utilisation que vous pouvez consulter en ligne.

https://apropos.erudit.org/fr/usagers/politique-dutilisation/ 


\section{REVIEW ESSAYS/NOTES CRITIQUE}

\section{Une Acadie à construire . . . mais où?}

« DEPUIS LA CONQUÊTE, L'ACADIE n'est plus une réalité politique ${ }^{1}$ ». C'est en 1957, peu de temps après les commémorations du bicentenaire du Grand Dérangement, que le révérend père Clément Cormier (1910-1987), éducateur et militant nationaliste acadien, décrivait la donne fondamentale de cette collectivité francophone dépourvue d'un État mais possédant bel et bien une vie nationale. Sans doute aurait-il été plus juste d'affirmer que la réalité acadienne n'avait plus d'assise géopolitique : en effet, depuis le milieu du $19^{\mathrm{e}}$ siècle, un projet collectif francophone était porté par la société civile, constituée d'institutions relativement autonomes et appuyée en grande partie par l'Église catholique. Il y avait donc une intention politique encadrée par une « élite définitrice ${ }^{2} \gg$ dont faisait partie l'orateur, qui prenait la parole à l'occasion d'un congrès destiné à moderniser la Société nationale l'Assomption, organisme de défense des intérêts acadiens, qui devenait dès lors la Société nationale des Acadiens (SNA)

Or, au moment où cette observation fut prononcée, la « petite nation ${ }^{4}$ » de l'Acadie des Maritimes était sur le point de connaître des mutations dont les effets se font sentir encore de nos jours. Au Nouveau-Brunswick, les réformes mises en œuvre par le gouvernement Robichaud allaient opérer « une vaste expropriation étatique du tissu social acadien ${ }^{5} »$. Par la suite, les populations francophones de

1 Clément Cormier, «La Société Nationale des Acadiens : préambule aux statuts et règlements », L'Évangéline, 24 juin 1957, p. 4.

2 Sur l'élaboration de ce concept en contexte franco-canadien et acadien, voir Jean-Paul Hautecœur, L'Acadie du discours : pour une sociologie de la culture acadienne, Québec, Presses de l'Université Laval, 1975, p. 22-23, 112, 207, 216-217; Fernand Dumont, Genèse de la société québécoise, Montréal, Boréal, 1993, p. 207-208; Marcel Martel, Le deuil d'un pays imaginé : rêves, luttes et déroute du Canada français. Les rapports entre le Québec et la francophonie canadienne (1867-1975), Ottawa, Presses de l'Université d'Ottawa, 1997, p. 43, 139, coll. " Amérique française »; Joel Belliveau, Le " moment 68 » et la réinvention de l'Acadie, Ottawa, Presses de l'Université d'Ottawa, 2014, p. 66, 260.

3 L'organisme s'appelle aujourd'hui la Société nationale de l'Acadie. Pour un historique de son évolution, voir Maurice Basque et Éric Snow, La Société nationale de l'Acadie : au cœur de la réussite d'un peuple, Moncton, Éditions de la Francophonie, 265 p.

4 Patrick D. Clarke explique dans les termes suivants, empreints de lyrisme, l'adéquation de ce qualificatif au cas acadien dans une conférence prononcée en 2009: "L'Acadie est une petite nation. Elle n'est pas que collectivité, et encore moins que simple minorité, que celle-ci soit juridique, ethnique ou associationnelle. Car elle n'est pas le fait du seul rapport social (ou encore du frottement des groupes) : elle est née de l'histoire et porte l'étendard d'une destinée. L'Acadie, c'est au fond un projet, un projet de faire histoire, voire même de faire société. Elle n'est pas d'abord un fait social, mais bien une entité nationale; elle n'est donc pas éphémère, mais ancrée dans le temps. » «Une foi raisonnée », discours prononcé le 17 août 2009 à la conférence « La pérennité du peuple acadien : défis et opportunités », parrainée par la Société nationale de l'Acadie dans le cadre du Congrès mondial acadien (CMA) de 2009, Tracadie-Sheila (N.-B.), http://snacadie.org/ sna/images/stories/documents/Une_foi_raisonne.pdf.

5 Greg Allain, Isabelle McKee-Allain et J. Yvon Thériault, « La société acadienne : lectures et conjonctures », dans Jean Daigle (dir.), L'Acadie des Maritimes : études thématiques des débuts à nos jours, Moncton, Chaire d'études acadiennes, 1993, p. 354-355.

Clint Bruce, « Une Acadie à construire . . mais où? », Acadiensis, vol. XLVII, n 2 (été/automne 2018), p. 129-141. 


\section{Acadiensis}

l'Atlantique n'ont pas échappé à la provincialisation de la francophonie canadienne qu'ont induite, d'une part, l'éclatement du Canada français, provoqué par la rupture entre le Québec et les communautés dites - pendant un certain un temps - « hors Québec », et, d'autre part, la politique biculturaliste du gouvernement fédéral. D'autres transformations sont liées à des tendances beaucoup plus englobantes. La solidarité ethnique cédant le pas à l'individualisation, il en aurait résulté, à en croire Joseph Yvon Thériault, "l'effondrement de l'Acadie politique comme lieu par excellence pour les Acadiens d'une mise en forme particulière de la coexistence humaine $^{6} »$. La logique néolibérale, rythmée par la mondialisation, aurait sapé la vitalité démocratique tout en diluant les repères identitaires, à telle enseigne que la conjoncture actuelle, que certains qualifient de «post-nationale ${ }^{7} »$, appellerait d'autres modalités de citoyenneté minoritaire, aux formes sans doute inédites. L'Acadie peut-elle se faire politique dans de telles conditions ?

Pour peu que l'on consente à accorder une certaine priorité à cette question, d'un point de vue ou scientifique ou citoyen, le moment est sans doute propice à un retour sur le chemin parcouru. Sous ce rapport, deux ouvrages récents ne sauraient manquer d'interpeller. Paru en 2015, L'Acadie politique : histoire sociopolitique de l'Acadie du Nouveau-Brunswick, de Michelle Landry, propose une synthèse critique des réflexions sur l'évolution de la collectivité acadienne dans la province où se concentre la grande majorité de la population francophone du Canada atlantique. Dans Audacieux et téméraire : le père Clément Cormier, c.s.s.(1910-1987), recteurfondateur de l'Université de Moncton, Marc Robichaud et Maurice Basque retracent la vie et la carrière d'une figure incontournable de la modernisation des institutions acadiennes au $20^{\mathrm{e}}$ siècle $^{8}$. À travers ces deux livres, l'un concis, l'autre volumineux, se donnent à lire des lectures à peu près compatibles, et assurément complémentaires, de la société acadienne. Certes, l'approche n'est pas la même : l'étude de Landry examine l'articulation entre discours et institutions, tandis que la biographie de Robichaud et Basque souligne l'influence des acteurs sur les institutions. D'un ouvrage à l'autre, ces chercheurs sont appelés à tracer les contours de l'entité sociétale, procédé qui produit et légitime l'objet étudié. C'est une opération fondatrice et fondamentale en sciences humaines, mais dont il ne faut jamais négliger les effets ${ }^{9}$. Après avoir signalé, dans un premier temps, les qualités

6 Joseph Yvon Thériault, L'identité à l'épreuve de la modernité : écrits politiques sur l'Acadie et les francophonies canadiennes minoritaires, Moncton, Éditions d'Acadie, 1995, p. 43.

7 Mireille McLaughlin, "L'Acadie Postnationale: Producing Franco-Canadian Identity in the Globalized Economy », thèse de Ph.D. (sociologie), Université de Toronto, 2010, 264 p.; Monica Heller, Paths to Post-Nationalism: A Critical Ethnography of Language and Identity, Oxford, Oxford University Press, 2011, 223 p.

8 Michelle Landry, L'Acadie politique : histoire sociopolitique de l'Acadie du Nouveau-Brunswick, Québec, Presses de l'Université Laval, 2015, 158 p., collection « Langues officielles et sociétés »; Marc Robichaud et Maurice Basque, Audacieux et téméraire : le père Clément Cormier, c.s.c. (1910-1987), recteur-fondateur de l'Université de Moncton, 2e édition revue et corrigée, Moncton, Institut d'études acadiennes, 2018, 433 p., collection « Mémoire biographique ». Une première édition, dans laquelle s'étaient glissées un certain nombre de coquilles, a été imprimée en novembre 2017.

9 Cf. Johannes Fabian, Time and the Other: How Anthropology Makes Its Object, New York, Columbia University Press, 1983, 205 p. 
respectives de ces deux contributions importantes aux études acadiennes, c'est sur ce travail de définition de l'Acadie, plus ou moins explicite, que nous porterons notre attention.

\section{Circonscrire l'Acadie politique}

Professeure de sociologie à l'Université de Moncton, Michelle Landry mène des recherches sur l'organisation sociopolitique de la communauté acadienne du Nouveau-Brunswick et plus largement sur les dynamiques de pouvoir se jouant autour des francophonies canadiennes ${ }^{10}$. Ses projets récents et actuels portent sur la gouvernance communautaire, la fonction mémorielle des manifestations culturelles et les enjeux de la municipalisation des régions rurales. Sa thèse de doctorat, soutenue en 2011 à l'Université Laval, sous la direction de Gilles Gagné11, est à l'origine de L'Acadie politique et le livre trouve tout à fait sa place dans la collection «Langues officielles et sociétés » des Presses de l'Université Laval. Dans le sillage de la pensée de l'historien Léon Thériault et du sociologue Joseph Yvon Thériault, c'est le problème de l'absence d'une emprise territoriale à même d'encadrer la nationalité acadienne qui oriente son exploration lucide, riche sur le plan théorique, des conditions changeantes du projet de société, à la fois fragile et animé d'un élan irrépressible, de « la communauté linguistique française du Nouveau-Brunswick », pour reprendre la dénomination juridique. Cette volonté de circonscrire la dimension politique de l'expérience acadienne, Landry la place sous le mot d'ordre d'un autre maître à penser, Fernand Dumont : «C'est entendu, on ne comprend l'état présent d'une société qu'en remontant à son passé12 ».

Sa lecture du moment présent est exposée dans la conclusion de l'ouvrage. Par contraste avec les stratégies dominantes des époques précédentes, au cours desquelles « la quête collective de reconnaissance orientée vers l'ensemble institutionnel plus vaste (Église, gouvernement, économie de marché, tribunaux, etc.) [...] s'est structurée autour d'associations et d'institutions » gérées par l'élite, la période contemporaine se caractérise par « la fragmentation de la cohérence d'ensemble » (p. 146). Ainsi, Landry en conclut à « l'incapacité des organismes et des institutions d'offrir des repères [afin] de structurer le vivre ensemble » tandis que « [1] e changement social, la contestation du statu quo, s'opère en effet à la fois

10 Parmi ses travaux déjà parus, on lira avec intérêt les articles suivants : Michelle Landry, «Esquisse d'une genèse de la société acadienne », Recherches sociographiques, vol. LIV, n 2 (2013), p. 305-323; Michelle Landry, « Se gouverner sans État ni territoire? Le cas des Acadiens du Nouveau-Brunswick », Cahiers du MIMMOC (Mémoire(s), identité(s), marginalité(s) dans le monde occidental contemporain), no 11 (2014), http://journals.openedition.org/mimmoc/1568, ou encore Michelle Landry, «Pour en finir avec le passage à la modernité des francophonies canadiennes », dans Michelle Landry, Martin Pâquet et Anne Gilbert (dir.), Mémoires et mobilisations, Québec, Presses de l'Université Laval, 2015, p. 221-237.

11 Michelle Landry, «La question du politique en Acadie : les transformations de l'organisation sociopolitique des Acadiens du Nouveau-Brunswick », thèse de doctorat (sociologie), Québec, Université Laval, 2011. Signalons que, à la différence de l'ouvrage traité ici, le titre de cette thèse adresse un clin d'œil à l'essai de Léon Thériault, défenseur d'une autonomie politique pour l'Acadie néo-brunswickoise : La question du pouvoir en Acadie, Moncton, Éditions d'Acadie, $1982,250 \mathrm{p}$.

12 Dumont, Genèse de la société québécoise, p. 9, cité dans Landry, L’Acadie politique, p. 1. 


\section{Acadiensis}

dans le quotidien et dans les coulisses du pouvoir » (p. 146). C'est en vue de rendre compte de ces développements que Landry cerne six périodes, examinées en autant de chapitres, à partir de la « Renaissance acadienne », dont les débuts coïncident avec la Confédération canadienne. Par ailleurs, aussi bien le dire tout de suite : la limpidité de l'argumentaire, porté par son organisation chronologique et le style accessible de l'auteure, le rend particulièrement apte aux besoins de l'enseignement; un séminaire sur la francophonie canadienne ou sur les minorités ethnolinguistiques s'en trouverait grandement enrichi.

L'Acadie politique tente (et réussit à plusieurs égards) un pari méthodologique et disciplinaire de la part de Landry : étudier l'histoire d'un peuple sans faire œuvre d'historienne, c'est-à-dire en se fiant à la littérature secondaire et en dépendant des interprétations d'autres chercheurs, du moins dans un premier temps. Justifiée en début de parcours ${ }^{13}$, cette démarche tient sa promesse en ce qui concerne son objectif d'analyser « comment l'organisation et les projets politiques des Acadiens du Nouveau-Brunswick se sont transformés et renouvelés » (p. 7). S'ancrant dans la sociologie des idéologies et des représentations, l'enquête de Landry privilégie davantage la superstructure - dont la manifestation pertinente est la référence, selon le concept de Dumont - que les conditions matérielles ayant conditionné l'émergence de cette dernière. Dans cette optique, l'an 0 , c'est 1763 - après la fin des déportations - et la véritable genèse de la société acadienne se déroule pendant les années 1860-1880. L'auteure s'abstient donc de s'aventurer sur le terrain des débats sur l'ethnogenèse acadienne pendant la période coloniale et le Grand Dérangement, non pas parce qu'elle les ignore mais plutôt parce que sa propre position en évince la pertinence ${ }^{14}$. Toujours à l'instar de Joseph Yvon Thériault, elle considère qu'il n'y pas de société acadienne sans référence symbolique. La construction de celle-ci participe de "l'édification d'une nation distincte », processus décrit dans le premier chapitre («La définition de la nation »). À la suite de la création des premières institutions éducatives et médiatiques à l'époque de la Confédération, la première Convention nationale acadienne, en 1881, affirme « la capacité des Acadiens de se définir collectivement, ainsi que leur désir de s'organiser et d'avoir prise sur leur histoire » (p. 38). Cette volonté est certes médiatisée par des discours exogènes, notamment les écrits de l'Américain Longfellow et du Français Rameau de Saint-Père. Toujours est-il que l'adoption de symboles nationaux témoigne d'une appropriation véritable de la part de la nouvelle élite définitrice.

La fin du $19^{\mathrm{e}}$ siècle et les premières décennies du $20^{\mathrm{e}}$ siècle voient des progrès en faveur d'« $[u]$ ne timide institutionnalisation » (titre du second chapitre) : régularisation des conventions nationales, fondation de deux organismes à vocation nationaliste (la Société l'Assomption et la Société nationale l'Assomption, dont la ressemblance des noms influera sur le changement voté en 1957), développement de

13 Landry précise que « cet ouvrage n'est pas à proprement parler une investigation historique, en ce sens qu'il repose surtout sur des sources secondaires. Bien qu'il prenne la forme d'une synthèse historique, la démarche est plus sociopolitique, c'est-à-dire qu'elle relève d'une perspective d'analyse qui met l'accent sur les rapports entre la société civile et l'État, mais également sur toutes les autres relations de pouvoir au sein des sociétés » (p. 4).

14 Landry évoque brièvement les arguments entourant l'ethnogenèse acadienne dans son article de 2013, « Esquisse d'une genèse de la société acadienne », p. 306-308. 
la presse et présence accrue au sein de la hiérarchie ecclésiastique. Les gains en matière d'éducation laissent cependant à désirer. C'est aux initiatives autour de ce dossier qu'est consacrée une partie du chapitre 3, «L'ère de l'éducation... et du clergé ». En termes de son argumentation, c'est peut-être le chapitre le moins efficace car, pour rendre compte des changements majeurs de la période 1920-1955, Landry doit embrasser du même coup d'œil plusieurs phénomènes concurrents comme la campagne pour la colonisation des terres, l'émergence du mouvement coopératif et l'arrivée dans les Maritimes de l'Ordre de Jacques-Cartier, société secrète vouée à la promotion sociale des Canadiens français. On a l'impression d'un passage obligé pour arriver au plus pressé au double bouleversement étudié dans le quatrième chapitre, "Étatisation et éclatement du discours », autrement cohérent sans sacrifier la concision voulue. Il s'agit justement de rendre compte de la transition de "l'Acadie politique », expression que Joseph Yvon Thériault réserve à la primauté de l'appartenance ethnique et de la référence nationaliste comme principe organisateur de la vie collective ${ }^{15}$, vers l'intégration à la société globale comme résultante de l'interventionnisme de l'État providence. C'est l'ère des refontes et des refondations des institutions, confrontées aux réformes du programme Chances égales pour tous. C'est aussi l'occasion, pour la génération montante, de contester le nationalisme traditionnel, irruption que Landry explique principalement en se référant à quelques travaux sur les mouvements étudiants et néonationalistes bien connus des acadianistes. Solidement articulés les uns aux autres, ces éléments sont exposés sans s'attarder outre-mesure sur la dimension de la référence mise de l'avant d'entrée de jeu ${ }^{16}$.

Les cinquième et sixième chapitres saisissent à bras-le-corps les dynamiques qui se sont installées au cours des années 1970-1980, dans un premier temps, et depuis l'avènement des Congrès mondiaux acadiens, dont le premier s'est déroulé en 1994, dans un deuxième temps. Dans « Face à l'État » (chap. 5), Landry prolonge la thèse de l'anthropologue Louis Cimino, qui a démontré que la reconfiguration du nationalisme ethnique dans les années 1960 représentait une réponse à la montée de l'État-providence ${ }^{17}$. En appliquant une analyse similaire aux effets de la Loi sur les langues officielles (1968) et de la reconnaissance de l'égalité des francophones au sein de la province désormais officiellement bilingue, égalité consacrée par la loi 88 (1981) avant d'être enchâssée en 1988 dans la Charte canadienne des droits et libertés, l'auteure rend compte des stratégies préconisées par le Parti acadien (1972-1982) et par la Société des Acadiens du Nouveau-Brunswick, créée en 1973. Elle résume (p. 118) :

Les Acadiens du Nouveau-Brunswick se sont dotés des outils qui leur semblaient nécessaires pour négocier avec l'État dans ce nouveau contexte. En encastrant les pratiques dans le territoire,

15 Joseph Yvon Thériault, «L'Acadie politique et la politique en Acadie », dans Thériault, L'identité à l'épreuve de la modernité, p. 29-50.

16 Pour ce qui des symboles acadiens, Landry cite une résolution qui fut adoptée lors du Ralliement de la jeunesse acadienne en avril 1966 et qui voulait reléguer le drapeau, l'hymne national et les autres « signes patriotiques » au domaine strictement patrimonial (p. 82).

17 Louis F. Cimino, Ethnic Nationalism Among the Acadians of New Brunswick: An Analysis of Ethnic Political Development, thèse de doctorat (anthropologie), Durham, Université Duke, 1977. 


\section{Acadiensis}

c'est-à-dire en définissant les organismes et les institutions selon les frontières politiques (provinciales), elles semblent alors s'être politisées et dé-ethnicisées. Ces «nouveaux » moyens institutionnels et organisationnels sont alors devenus les nouveaux repères de leur définition et la politique tout comme les tribunaux ont été ajoutés au répertoire des stratégies possibles et des leviers de pouvoir.

Les conditions sont donc réunies pour que l'auteure jette un regard critique sur la résurgence du discours ethnicisant au tournant des années 1990, sujet du chapitre joliment intitulé « Entre prise de parole et prise de sang ». À ce moment-là, les Acadiens du Nouveau-Brunswick ne semblent plus se résoudre au simple statut d'une « communauté linguistique » détachée de ses extensions diasporiques du fait du Grand Dérangement et des migrations depuis le $19^{\mathrm{e}}$ siècle. Y a-t-il contradiction? C'est autour de cette question que Landry formule quelques-unes de ses observations les plus perspicaces et les plus originales. Cependant, elle tient à l'écart certains aspects de ce phénomène qui ne cadrent pas avec l'opposition qu'elle dresse entre une vision diasporique et une conception sociopolitique de l'Acadie : y échappent notamment les efforts pour concilier ces deux pôles par une image inclusive et multiculturelle de la société acadienne lors du CMA de 200918, ou encore les expériences ni moins pertinentes ni moins «politiques » des communautés francophones de la Nouvelle-Écosse et de l'Île-du-Prince-Édouard. C'est sur ce deuxième point que je reviendrai plus tard.

\section{Un bâtisseur sur le chantier de l'extrême frontière}

L'œuvre inlassable de Clément C. Cormier - de qui Landry signale l'influence auprès du premier ministre Louis J. Robichaud dans la création de l'Université de Moncton (p. 74) - mérite sans doute, comme le souhaitait Théo Nkembé en signant une première biographie parue en 1979, toujours du vivant de son sujet, que lui soient consacrées « des biographies beaucoup plus approfondies de celui qui, pendant toute sa vie, a fièrement porté les couleurs acadiennes dans la lutte qui fut celle de la Renaissance de tout son peuple ${ }^{19} »$. L'envol dithyrambique ne devrait pas éclipser la justesse du propos. Religieux de la congrégation de Sainte-Croix et élève de Georges-Henri Lévesque à l'École des sciences sociales de l'Université Laval, « le père Clément » devient une figure de proue de «l'idéologie de la participation modernisatrice » (la formule est de Ricky Richard ${ }^{20}$ ) en Acadie du Nouveau-

18 Les efforts et débats en vue de présenter une Acadie « diverse » et « ouverte sur le monde » à l'occasion du CMA de 2009, qui s'est déroulé dans la région de la Péninsule acadienne, au Nouveau-Brunswick, ont été traités par Marie Lefebvre, « Le Congrès mondial acadien : entre mémoire et territoire », Études canadiennes/Canadian Studies, no 69 (2010), p. 53-68, et par Monica Heller, Lindsay A. Bell, Michelle Daveluy, Mireille McLaughlin et Hubert Noël dans Sustaining the Nation: The Making and Moving of Language and Nation, New York, Oxford University Press, 2015, 288 p.

19 Théo Nkembé, Père Clément Cormier, fondateur en Acadie, Moncton, s.n., 1979, p. 142.

20 Cité par Joel Belliveau dans « Le père Clément Cormier et l'âge d'or du paradoxal "nationalisme libéral" en Acadie du Nouveau-Brunswick, 1945-1967 », dans Frédéric Boily et Donald Ipperciel (dir.), D’une nation à l'autre : discours nationaux au Canada, Québec, Presses de l'Université Laval, 2011, p. 225, collection «Prisme». 
Brunswick. L'éducation représente son principal cheval de bataille : après avoir enseigné et servi d'administrateur au sein de l'Université Saint-Joseph de Memramcook, dont il sera recteur de 1948 à 1954 et de 1956 à 1963, il se fera le principal artisan de la transformation de l'Université Saint-Joseph et de la fondation de l'Université de Moncton, où sa statue, créée par l'artiste Claude Roussel, accueille aujourd'hui les visiteurs devant l'entrée de la bibliothèque Champlain. C'est là le fil conducteur de l'imposante biographie, méticuleusement et copieusement documentée par Marc Robichaud et Maurice Basque, de cet ecclésiastique à l'esprit moderne dont l'implication s'étend à " presque toutes les causes et tous les dossiers acadiens » (p. 2) entre les années 1940 et 1970. Récit d'une grande finesse, Audacieux et téméraire vient s'ajouter à la collection «Mémoire biographique » de l'Institut d'études acadiennes (IEA) de l'Université de Moncton.

Écrire une telle biographie n'a rien d'une mince affaire, et celle-ci, de près de 400 pages, n'a pas la mine d'une mince plaquette. Le défi consistait à rendre justice à un acteur majeur d'initiatives aussi diverses que les commémorations du bicentenaire de la Déportation et la Commission royale d'enquête sur le bilinguisme et le biculturalisme, en passant par d'innombrables services rendus aux organismes de la société civile, sans succomber à la dispersion. Certes, Robichaud et Basque étaient on ne peut mieux placés pour s'attaquer à ce projet, d'autant plus que les deux historiens n'en sont pas à leur première collaboration. Chercheur autonome lors de la parution de l'ouvrage, Robichaud a été affilié pendant plusieurs années à l'IEA, que Basque a dirigé de 2007 à 2010 après avoir administré, une dizaine d'années durant, le Centre d'études acadiennes (CEA). (Celui-ci fut fondé par le père Cormier en 1968.) De toute évidence, leur travail autour du beau-livre Histoire de l'Université de Moncton (2013) aura préparé le terrain ${ }^{21}$.

L'ampleur de la documentation aura exigé des efforts de taille, lesquels se traduisent notamment par la création d'une bibliographie des écrits de Cormier, beaucoup non signés, dont le résultat final est disponible en ligne ${ }^{22}$. Plusieurs sources permettent de nuancer le statut quasi mythique du personnage; à côté des correspondances et des journaux intimes, les auteurs exploitent une série d'entretiens avec des proches du père Cormier, réalisés par Robert Pichette. Alors que la «panthéonisation » du père Cormier s'est surtout cristallisée autour de son rectorat, la période suivant la création de l'Université de Moncton n'est traitée que dans le cinquième et dernier chapitre (qui fait quand même une centaine de pages!). Jusque-là, il s'agit surtout de la genèse de cette initiative et des influences et expériences qui l'ont préparée.

21 Leur Histoire de l'Université de Moncton (Moncton, Institut d'études acadiennes, 2013, 383 p.) a été précédée d'un ouvrage co-écrit avec Sylvie Ladouceur, Vivre sa santé en français au NouveauBrunswick : le parcours engagé des communautés acadiennes et francophones dans le domaine de la santé (Moncton, Institut d'études acadiennes, 2011, 103 p.).

22 Cette bibliographie, d'une indéniable utilité pour d'autres chercheurs, représente la continuation d'un projet amorcé par Nkembé dans les années 1970. Marc Robichaud et Maurice Basque, Clément Cormier, c.s.c. : écrits, publications et travaux, Moncton, Institut d'études acadiennes, 2017, http://www.umoncton.ca/iea/files/iea/wf/wf/images/publications_et_ecrits_du_pere_clement_ cormier.pdf. 


\section{Acadiensis}

La recherche contemporaine cautionne pleinement cette approche. Dans un article de 2009, "Portrait d'un "fondateur dans l'âme" », le sociologue Julien Massicotte fait voler en éclats l'idée reçue selon laquelle l'apparition des sciences sociales se serait produite par génération spontanée au début des années $1960^{23}$. L'impératif d'humaniser le capitalisme tout en tenant en échec les idéologies marxistes, double préoccupation déjà présente dans le mouvement coopératif, anime également le catholicisme social dont se nourrira l'esprit de Cormier lors de son passage à l'École des sciences sociales de l'Université Laval, sous la direction de Georges-Henri Lévesque. C'est sous l'influence de la " sociologie de l'ordre » qu'est fondée, dès 1939, l'École des sciences sociales de l'Université Saint-Joseph de Memramcook, initiative qui permet à Cormier de déployer une pédagogie dynamique et novatrice qui, soupçonne Nkembé, « devait gêner bon nombre de ses collègues $^{24} »$. Sur les traces de Massicotte, Joel Belliveau démontre à quel point le terrain idéologique des réformes de l'ère Robichaud a été préparé par les « réformateurs frustrés » des années 1950 : «Grâce au travail acharné de la part de la frange la plus progressiste de l'élite définitrice », affirme l'historien au sujet du courant dont Cormier fut le moteur, "l'idéal du libéralisme est implanté dans la psyché acadienne » dès avant les élections de 1960. Cette transformation aurait porté fruit dans la mesure où, désormais, « [1]'Acadie [était] prête pour la modernité et pour l'intégration dans le monde plus large $»^{25}$. Bien qu'il appartienne à « l'élite cléricale », Clément Cormier n'a donc rien d'un ultramontain rigoriste. Tout au contraire, c'est un désir de progrès collectif battant la cadence de la marche du monde, qui caractérise la perspective de ce natif de Moncton, bon vivant à l'énergie expansive dont le «zèle s'est exercé dans tous les domaines et dans toutes les questions sociales qui ont façonné l'Acadie contemporaine ", comme l'a déjà rappelé Robert Pichette ${ }^{26}$.

En amont des appréciations de ces chercheurs, force est de compter avec l'analyse livrée par Jean-Paul Hautecœur dans L'Acadie du discours : pour une sociologie de la culture acadienne (1975), ouvrage aussi incisif qu'incontournable. À l'époque où le sociologue français, chassé de l'Université de Moncton après les soubresauts de 1968-1969, faisait publier sa thèse de doctorat, Clément Cormier venait de quitter la direction du Centre d'études acadiennes pour devenir chancelier de l'Université. On pourrait penser qu'un tel poste honorifique lui conférerait plus de prestige, sinon de pouvoir, que celui qu'il délaissait. Pourtant, c'est plutôt grâce à ses écrits dans Les Cahiers de la Société historique acadienne que Hautecœur voit

23 À cet égard, Massicotte écrit : «L'étude de la fondation et des premières orientations de l'École des sciences sociales de l'Université Saint-Joseph de Memramcook est importante et essentielle même pour comprendre l'essor politico-institutionnel de l'Acadie du Nouveau-Brunswick dans les années 1960 et la modernisation de l'État provincial. Cette composante est trop peu abordée par les analystes; trop souvent on considère la "modernité" acadienne comme née en 1960, alors que rien n'est plus faux. » Julien Massicotte, « Portrait d'un "fondateur dans l'âme" : Clément Cormier, pionnier des sciences sociales en Acadie du Nouveau-Brunswick », Acadiensis, vol. XXXVIII, no 1 (hiver/printemps 2009), p. 31.

24 Nkembé, Père Clément Cormier, p. 36.

25 Belliveau, « Le père Clément Cormier et l'âge d'or », p. 225.

26 Robert Pichette, " "Longtemps l'Acadie a attendu un chef" : Clément C. Cormier, c.s.c. (19101987) », Cahiers de la Société historique acadienne, vol. 30, nº 4 (décembre 1999), p. 238. 
en son ancien patron « un maître-idéologue 27 ». Alors que pour Belliveau les réformateurs regroupés autour de Cormier se voulaient « avant tout des hommes d'action, plus préoccupés par la gestion d'organisations que par les discours sur la nation ${ }^{28}$ », Hautecœur tient le père Cormier pour « l'un des principaux définiteurs du nationalisme acadien contemporain ${ }^{29} »$. Nonobstant ses velléités de progressisme et son inlassable activité pour la modernisation de l'Acadie, l'auteur lui attribue un conservatisme discursif qui osait si peu dévier du pieux plain-chant truffé de versets composés lors des Conventions nationales. Même en plein 20e siècle, l'impératif aurait consisté à affirmer « la bienheureuse totalité de la communauté traditionnelle » afin de conserver à l'idéologie nationaliste « son pouvoir de totalisation ». À en croire Hautecœur, le père Cormier aurait, à lui seul, incarné ce rôle-là : «Ce qu'il est important de retenir, juge-t-il sans fléchir, c'est que l'homme figure là où la nation est personnellement représentée ${ }^{30} »$. Ainsi, Clément Cormier est entièrement assimilé à son discours.

Sans tâcher de réfuter l'analyse de Hautecœur, Audacieux et téméraire adopte une perspective tout autre ${ }^{31}$. Derrière l'Acadie du discours, il y a l'Acadie du vécu. C'est dans cette Acadie-là, dynamique et en pleine évolution au tournant du $20^{\mathrm{e}}$ siècle, que Robichaud et Basque nous plongent afin de cerner les facteurs socioculturels qui vont façonner la pensée et l'agir du futur « fondateur ». Le premier chapitre, « Deux familles, une ville », dépeint un milieu familial défini par la mobilité et l'univers urbain, sans que les liens avec la campagne ne soient entièrement rompus. Son grand-père ayant émigré en Nouvelle-Angleterre dans les années 1870, c'est au Massachusetts que naît en 1885 le père de Cormier, Clément J. Cormier, avant de grandir chez son oncle à Cocagne et, vers l'âge de 20 ans, de déménager à Moncton. Née et élevée à Memramcook, Léontine Breau s'installe dans la même ville, où elle exerce le métier de couturière au moment où elle épouse Clément J. Cormier en 1907. Tandis que l'essor économique de Moncton attire un nombre grandissant d'Acadiens et d'Acadiennes, les institutions francophones s'y implantent durablement : le journal L'Évangéline en 1910, la Société 1'Assomption, fondée au Massachusetts en 1903, y établit son siège social en 1913. Le milieu socioculturel de l'enfance de Clément Cormier est marqué aussi bien par l'implication de ses parents dans les associations acadiennes que par la volonté de se tailler une place au sein de la société dominante : le deuxième chapitre, « De Moncton à Memramcook : la jeunesse de Clément Cormier », examine donc l'intersection des questions nationales avec le monde anglophone. Par exemple, Clément J. Cormier, « pilier du mouvement associatif acadien » (p. 75) et secrétairetrésorier et gérant du journal libéral L'Acadien, est élu au conseil municipal de Moncton en 1923.

27 Hautecœur, L'Acadie du discours, p. 41.

28 Belliveau, «Le père Clément Cormier et l'âge d'or », p. 218.

29 Hautecœur, L'Acadie du discours, p. 41.

30 Hautecœur, L'Acadie du discours, p. 154.

31 Robichaud et Basque ne manquent pas de souligner que les écrits du père Cormier dans Les Cahiers de la Société historique acadienne "sont soumis à une critique très sévère véhiculée dans les années 1960 par les fondateurs » de cette société, qui comptent, aux côtés de Cormier, le R. P. Anselme Chiasson et Emery LeBlanc (p. 5). 


\section{Acadiensis}

L'exploration de cet environnement permet également de faire valoir une autre influence intellectuelle, celle des femmes instruites et engagées. En tant que préfet des études de l'Université Saint-Joseph, phase de sa carrière abordée dans le troisième chapitre ( «Prêtre, professeur et préfet des études »), le père Cormier fera de l'accès équitable à l'éducation l'un de ses chevaux de bataille (p. 184-190) ${ }^{32}$. Ce principe-là, qui ne va pas sans controverse, orientera aussi sa volonté d'intégrer à la nouvelle Université de Moncton les étudiantes du Collège Notre-Dame d'Acadie de Moncton, qui fermera ses portes en 1965.

Dans un poème composé en 1981, l'écrivain Gérald Leblanc évoque, au détour d'un vers qui renferme le titre d'un recueil, « la réserve dont Moncton est l'extrême frontière ${ }^{33} »$. Se réclamer de cette ville et y revendiquer un lieu de vie collective revient à récuser une vision communautariste, fondée sur la partition ethnique ${ }^{34}$. Audacieux et téméraire s'efforce de témoigner de cette idée-force du père Cormier : s'il y avait une Acadie moderne à bâtir, Moncton devait en être le chantier. Il importait de « conquérir la ville». C'est donc vers une concentration des institutions acadiennes qu'ont tendu bon nombre des efforts qu'il a menés, tels qu'exposés par Robichaud et Basque : l'ouverture du campus monctonien de l'Université SaintJoseph en 1953, suivie du déménagement définitif de celle-ci, l'établissement de la radio et de la télévision francophones de Radio-Canada, la «monctonisation » de la Société nationale des Acadiens au début des années $1960^{35}$ et la configuration unifiée qu'il souhaitait donner à l'Université de Moncton. La vision centralisatrice du père Cormier n'a pas fait que des heureux - loin de là. Toutefois, il aura plus tard, après le compromis de 1975 établissant trois campus, l'impression que l'élan en faveur la cohésion nationale aura été sacrifié à un provincialisme tenace : «Amer d'avoir été écarté de la direction universitaire par la laïcisation de l'administration en 1967 et grand opposant de la formule trinitaire faisant de l'Université de Moncton, depuis 1977, une institution à trois campus égaux, le père Cormier, jusqu'à la fin, défendra sa vision d'une université centralisée » (p. 279). Pour Basque et Robichaud, le fondateur aurait été aveuglé par une profonde « méconnaissance des réalités régionales » (p. 283). De là un portrait presque tragique d'un homme qui a tant contribué : à l'instar du passage ci-dessus, les mots « amer » et « amertume » reviennent à plusieurs reprises lorsqu'il est question des dernières années de la vie de Cormier.

Audacieux et téméraire prend le contrepied de L'Acadie du discours à de nombreux égards, dont le moindre n'est pas de privilégier la parole intime. Les documents informels y ont autant de poids, sinon plus, que les discours, écrits

32 Comme préfet des études de l'Université Saint-Joseph, il se montre favorable aux activités mixtes et, de concert avec sœur Jeanne de Valois, veille à la création du premier cours classique pour filles.

33 Gérald Leblanc, L'extrême frontière : poèmes 1972-1988, Sudbury, Prise de parole, 2015, p. 75.

34 Joseph Yvon Thériault commente " la nostalgie de la "réserve" ", manifestation d'un pôle communautariste perceptible dans le discours néonationaliste des années 1970 et dans certaines réflexions contemporaines, dans un essai intitulé « Le triangle de l'acadianité », dans L'identité à l'épreuve de la modernité, p. 113-127.

35 Il s'agit d'une observation de Basque dans Basque et Snow, La Société nationale de l'Acadie, p. 123, cité dans Audacieux et téméraire, p. 269. 
publiés et autres manifestations de la parole publique. Certains témoignages mettent en lumière des excès de personnalité et un penchant marqué à mal étreindre à force de trop embrasser. Il lui fut notamment reproché ses fréquentes absences pendant son rectorat ou encore une assiduité moins qu'exemplaire en tant que membre de la Commission Laurendeau-Dunton. Même si les manifestations étudiantes de la fin des années 1960 sont peu traitées, les auteurs ne manquent pas de souligner le boycottage de l'investiture du père Cormier comme chancelier en 1973. Ces aspects de son parcours sont évoqués sans complaisance; effectivement, l'un des thèmes de l'ouvrage concerne la tension entre la cohérence de sa vision et la dispersion de ses intérêts et activités, ce qui a entraîné des problèmes de santé récurrents.

D'autres documents ne font que rehausser la touchante sincérité du sentiment patriotique qui l'aura guidé toute sa vie durant. En soupesant les avantages et les inconvénients de la carrière ecclésiastique qu'il a fini par choisir, le jeune Clément Cormier avait consigné l'aspiration suivante parmi les potentialités d'une vocation religieuse : «J'aurai une chance de travailler pour l'Acadie. (Une belle œuvre)» (p. 119).

\section{Le regard qui rapetisse, ou l'Acadie sous régime synecdochique}

Malgré leurs sujets et leurs objectifs très distincts, ces deux ouvrages récents se recoupent à plusieurs niveaux et se rejoignent à plusieurs égards. L'un de leurs dénominateurs communs consiste à assimiler, implicitement ou explicitement, l'Acadie moderne au Nouveau-Brunswick francophone. C'est à cela que rime le titre même de l'ouvrage de Landry, qui établit un rapport de consubstantialité entre «l'Acadie politique » et «l'Acadie du Nouveau-Brunswick». Même justifiée pour des raisons méthodologiques, l'imposition épistémologique des frontières provinciales opère trop souvent un découpage que le discours scientifique s'efforce de naturaliser en retranchant des excroissances d'acadianité.

Or, nous savons bien que la territorialité du fait acadien n'a rien d'aussi simple : c'est justement la dispersion des communautés acadiennes et d'origine acadienne problématique maintes fois constatée, commentée et étudiée - qui fait en sorte que toute tentative d'étudier l'Acadie doit, ce faisant, produire une définition de l'Acadie, qu'elle soit formulée en termes explicites ou sécrétée par les présupposés. Or, la tendance à la surlocalisation semble être lourde. Ainsi, un essai extrêmement éclairant de Patrick Clarke sur les « Régions et régionalismes en Acadie » aborde cette problématique sans évoquer les réalités de la Nouvelle-Écosse, de l'Île-duPrince-Édouard ou des Îles-de-la-Madeleine : par synecdoque, le NouveauBrunswick francophone se substitue à l'Acadie tout entière ${ }^{36}$. Tandis que des recherches récentes de Michael Poplyansky ont rappelé que les contestations étudiantes des années 1968 n'ont pas épargné le Collège Sainte-Anne en NouvelleÉcosse $^{37}$, Philippe Volpé a reproché à Joel Belliveau d'avoir minimisé leur portée dans les collèges du nord du Nouveau-Brunswick. Volpé s'est senti obligé de

36 Partick D. Clarke, «Régions et régionalismes en Acadie : culture, espace, appartenance », Recherches sociographiques, vol. 41, $\mathrm{n}^{\circ} 2$ (2000), p. 206-214.

37 Michael Poplyansky, en collaboration avec Mylène Comeau, "Le "moment 68" au Collège Sainte-Anne : la mentalité estudiantine au moment de la grève de 1968 », Historical Studies in Education/Revue d'histoire de l'éducation, vol. 30, nº 1 (printemps 2018), p. 114-140. 
souligner que " l'Acadie, ce n'est pas Moncton, non plus l'Université de Moncton $^{38} »$ (n'en déplaise au père Cormier).

Sa mise en garde fait drôlement écho à un éditorial paru en 1976, au moment où les intérêts divergents des associations provinciales menaçaient de fragiliser les relations entre l'ensemble des Acadiens: «Les Acadiens de la Nouvelle-Écosse et de l'Île-du-Prince-Édouard viennent de nous rappeler brutalement qu'ils sont membres à part entière de cette grande famille acadienne. Et cela, il ne faut jamais l'oublier : le peuple acadien ne se limite pas au Nouveau-Brunswick. Il faut résister à la tentation de nous replier sur nous-mêmes ${ }^{39} 》$. Au $21^{\text {e }}$ siècle, il se peut très bien, comme le fait observer Landry, que « les Acadiens [aient] trouvé une place assez confortable au sein de la société néo-brunswickoise [...] pour se permettre d'investir des ressources et de l'énergie dans la réanimation des mythes liés à la Déportation et à la filiation, plutôt que dans le développement de leur propre communauté politique » (p. 144-145). Malgré des gains considérables, les Acadiens des autres provinces ne bénéficient guère d'un tel « confort ». Parmi d'autres exemples possibles, la cause récente de la représentation effective en Nouvelle-Écosse rappelle à quel point le confort ressemble à un luxe hors Nouveau-Brunswick ${ }^{40}$.

Ces remarques ne comportent aucune critique intrinsèque à l'égard du livre de Robichaud et Basque. Bien sûr, les contributions de Clément Cormier n'étaient guère restreintes au Nouveau-Brunswick - bien loin de là. Il aurait été intéressant de faire comprendre davantage la dimension panacadienne et internationale de son implication, mais il faudrait sans doute qu'un autre ouvrage s'en charge. Toutefois, pour se faire une idée juste des champs d'intervention des promoteurs de l'Acadie modernisante, il me semble quand même pertinent d'accompagner la lecture d'Audacieux et téméraire d'une autre biographie, Le rassembleur : Léger Comeau (1920-1996), de Maurice Rainville et Simone LeBlanc-Rainville. Originaire du sudouest de la Nouvelle-Écosse et président de la Société nationale des Acadiens de 1978 à 1988, Léger Comeau présente de nombreux points de comparaison avec Clément Cormier. Lui aussi " homme de toutes les luttes », c'est également quelqu'un «qui se plaît surtout à construire »; " ses idées progressistes et ses critiques à l'endroit de l'Église » font obstacle à son élévation au rang d'évêque ${ }^{41}$. Le portrait du père Comeau fait état d'une trajectoire transprovinciale - non seulement il a vécu 17 ans au Québec, mais aussi il a résidé et œuvré au NouveauBrunswick - et d'une action tournée vers l'international, soit le renforcement des

38 Philippe Volpé, «Autour de l'éducation : plaidoyer pour une histoire incarnée de l'Acadie », Acadiensis, vol. XLIV, $\mathrm{n}^{\circ} 2$ (été/automne 2015), p. 131-148.

39 Cet éditorial de Claude Bourque, de L'Évangéline, a été reproduit dans Le Petit Courrier, aujourd'hui Le Courrier de la Nouvelle-Écosse. Je remercie Chantal White de l'avoir porté à mon attention. "Les Acadiens du N.-B. sont-ils renfermés sur eux-mêmes ? », Le Petit Courrier, 24 juin 1976, p. 15.

40 Voir le rapport et les recommandations de la Commission sur la représentation effective des électeurs acadiens et afro-néo-écossais, «Représentation : Pour une représentation effective des Acadiens et des Afro-Néo-Écossais », https://novascotia.ca/representation/RepresentationToward-More-Effective-Representation-For-Acadian-and-African-Nova-Scotians-Report-andRecommendation-fr.pdf.

41 Maurice Rainville et Simone LeBlanc-Rainville, Le rassembleur : Léger Comeau (1920-1996), Moncton, Éditions d'Acadie, 2000, p. 138, 305, 307. 
liens avec la France et l'ouverture vers la francophonie mondiale. Autant la carrière du père Cormier ne se limite pas à Moncton, autant celle du père Comeau implique également un engagement très fort envers des communautés locales. Mettre l'accent sur des forces d'attraction centripètes ou centrifuges, ce sont des façons complémentaires d'étudier la même société.

Chez Landry, le choix de privilégier le Nouveau-Brunswick relève d'une approche tout à fait justifiable. Avancer la spécificité néo-brunswickoise - compte tenu, parmi d'autres facteurs, de son poids démographique - aussi bien au sein de cette province que dans l'ensemble de la population acadienne des Maritimes -, de sa solidité institutionnelle et du régime de dualité linguistique dont il bénéficie en vertu de la Constitution canadienne - n'a rien d'illégitime. C'est justement ce à quoi s'efforce Léon Thériault dans son essai à saveur autonomiste La question du pouvoir en Acadie, lorsqu'il prétend qu'il ne peut y avoir un projet de société acadienne qu'au sein de la «Province pittoresque » (ou dans une province autonome détachée du Nouveau-Brunswick). Si la démarche de Landry s'inscrit peu ou prou dans cette optique, la revendication autonomiste en moins, et si L'Acadie politique tient si peu compte des communautés acadiennes des autres provinces, l'auteure semble avoir fait l'économie de toute justification de ce choix lors de la transformation de sa thèse de doctorat en livre. Par conséquent, ces autres communautés sont vouées à une sorte d'inexistence. Il est vrai qu'elle estime, dans une note de bas de page, que sa périodisation de l'histoire acadienne gagnerait à être confrontée aux développements en Nouvelle-Écosse et à l'Île-du-Prince-Édouard (p. 4). Mais dans l'ensemble, ces populations ne sont évoquées que pour affirmer qu'il apparaît « pratiquement impossible d'assurer une cohérence entre les Acadiens des trois provinces maritimes » (p. 90). Les tensions autour de cette nouvelle donne ne font pas l'objet d'un examen, comme si elles n'appartenaient pas au domaine de l'Acadie politique.

"Moins que des intentions personnelles, des localisations socioculturelles mobilisent alors l'intérêt et le type de recherche », affirme Michel de Certeau dans L'écriture de l'histoire ${ }^{42}$. Il y a lieu de s'interroger sur ce phénomène en études acadiennes : Où construit-on l'Acadie? Et en faisant abstraction de quelles dimensions de l'expérience acadienne? Peut-être parler de modernité acadienne ou de politique acadienne en faisant abstraction de leurs déploiements multiples? De Certeau précise : « Est abstraite, en histoire, toute "doctrine" qui refoule son rapport à la société. Elle dénie ce en fonction de quoi elle s'élabore. Elle subit alors les effets de distorsion dus à l'élimination de ce qui la situe en fait sans qu'elle le dise ou le sache : un pouvoir, qui a sa logique; un lieu, qui sous-tend et "tient" une discipline dans son déploiement en œuvres successives, etc. ${ }^{43}$ ». Les chercheurs, eux, se trouvent donc face à trois options : soit accorder une valeur téléologique à l'unicité du Nouveau-Brunswick en marginalisant ou en rendant invisible le reste de l'Acadie des Maritimes, soit tenir pour acquis que ces populations ont des existences parallèles, ou encore considérer que les interactions, les frictions et les fins de non-recevoir entre elles constituent une problématique fondamentale de l'Acadie politique.

\section{CLINT BRUCE}

